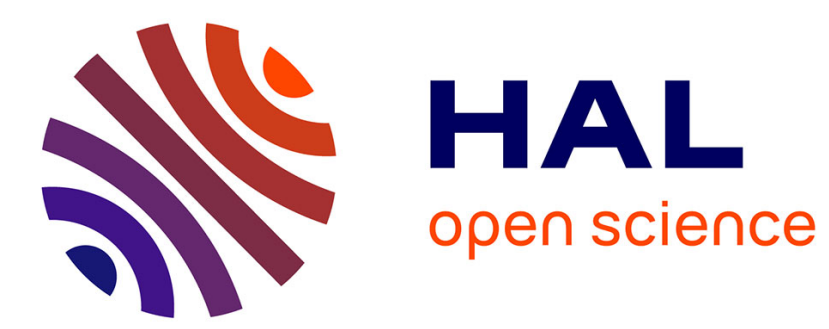

\title{
Fibre gratings for hydrogen sensing
}

Alain Trouillet, Emmanuel Marin, Colette Veillas

\section{To cite this version:}

Alain Trouillet, Emmanuel Marin, Colette Veillas. Fibre gratings for hydrogen sensing. Measurement Science and Technology, 2006, 17 (5), pp.1124-1128. 10.1088/0957-0233/17/5/S31 . ujm-00104535

\section{HAL Id: ujm-00104535 \\ https://hal-ujm.archives-ouvertes.fr/ujm-00104535}

Submitted on 6 Oct 2006

HAL is a multi-disciplinary open access archive for the deposit and dissemination of scientific research documents, whether they are published or not. The documents may come from teaching and research institutions in France or abroad, or from public or private research centers.
L'archive ouverte pluridisciplinaire HAL, est destinée au dépôt et à la diffusion de documents scientifiques de niveau recherche, publiés ou non, émanant des établissements d'enseignement et de recherche français ou étrangers, des laboratoires publics ou privés. 


\title{
Fibre gratings for hydrogen sensing
}

\author{
Alain Trouillet, Emmanuel Marin and Colette Veillas \\ Laboratory of Signal Processing and Instrumentation, UMR CNRS 5516, \\ Jean Monnet University, 18, rue du Pr Benoît Lauras, F-42000 Saint-Etienne, France \\ E-mail: alain.trouillet@univ-st-etienne.fr
}

Received 13 July 2005, in final form 7 December 2005

Published 13 April 2006

Online at stacks.iop.org/MST/17/1124

\begin{abstract}
Liquid hydrogen has been intensively used in aerospace applications for the past 40 years and is of great interest for future automotive applications.

Following major explosive risks due to the use of hydrogen in air, several studies were carried out in order to develop optical fibre sensors for the detection of hydrogen leakage. This paper aims at the presentation of new hydrogen sensors based on the use of fibre Bragg gratings (FBG) and long period gratings (LPG) coated by palladium nanolayers. The sensing principle based on the palladium-hydrogen interaction is presented, as well as experimental results. It is shown that both techniques could be used for hydrogen sensing but with a sensitivity enhanced by a factor up to 500 when using a LPG sensor. FBG sensors appear to be pure strain sensors and LPG sensors are mainly based on the coupling between the cladding modes and evanescent or surface plasmon waves. Preliminary results obtained with an in-fibre Mach-Zehnder interferometer configuration with in-series LPG sensors are also presented. They show potential interest to compensate for the thermal sensitivity of the fibre gratings.
\end{abstract}

Keywords: optical fibre sensors, hydrogen detection, fibre Bragg gratings, long period gratings

(Some figures in this article are in colour only in the electronic version)

\section{Introduction}

Due to the well-known explosive risks of hydrogen systems, hydrogen sensors are of great interest for both aerospace applications and future developments in the fields of fuel cells and automotive applications. A great number of hydrogen sensors [1, 2] have been demonstrated and merchandized in the past 20 years. Even though they were not specific to hydrogen detection, hydrocarbon sensors have often been used. Most of them provided fast and efficient detection at room temperature and pressure, but their principles and conceptions were not adapted for applications where the need for a remote local detection in a harsh atmosphere leads to the choice of optical fibre sensors. Among the numerous methods for gas detection two very different principles have been used for hydrogen detection. They are based on spectroscopic analysis via infrared absorption or Raman scattering [3, 4] for the first one and interaction with a transducer which has a thin palladium layer [5] in most cases. Several methods have been demonstrated; the most significant were based on surface plasmon resonance [6], intensity variations by means of electrochromatic materials $[7,8]$ or interferometric measurements [9]. Following the works of Butler [10, 11] studies have been carried out at the University of Saint-Etienne in order to develop optical fibre sensors for the detection of hydrogen leakage. The most relevant of them were based on a nanometric palladium micro-mirror deposited on the cleaved output end face of a multimode optical fibre [12] or based on surface plasmon resonance at the interface between the bare core of a multimode optical fibre and a deposited palladium nanolayer [13]. All the prototypes and developed sensors were based on the monitoring of intensity variations of a monochromatic light propagated through the optical fibre and the potentialities of the SPR sensors for volumetric detection have also been demonstrated.

Nevertheless, the requirements of the next generation of sensors must also be considered [14]. In addition to improve functionality at a lower cost, the new sensors, regardless of operating technology, must be configured in networks providing global information from real time data. Due to 
their addressing capabilities, the use of fibre Bragg gratings (FBG) and long period gratings (LPG) could contribute to the development of such a new sensor generation. LPG have already been used successfully as solution concentration sensors where the cladding modes interacted with the solution into which the sensor was immersed $[15,16]$. We present optical fibre hydrogen sensors based on FBG and LPG on the surface of which a palladium nanolayer is deposited. Preliminary results obtained with an in-fibre Mach-Zehnder interferometer configuration with in-series LPG sensors are also presented.

\section{Principle of operation}

FBG and LPG are intrinsic devices that allow for the control of the properties of the light propagated inside the fibre. They generally consist of a periodic modulation of the refractive index of the optical fibre core. The presence of the fibre grating promotes the coupling of the fundamental guided mode in the core with forward or backward modes. The coupling is accomplished under the phase matching condition (1) [15]:

$$
\lambda=\left[n_{\mathrm{eff}}^{\mathrm{o}}(\lambda)-n_{\mathrm{eff}}^{i}(\lambda)\right] \Lambda
$$

where $n_{\text {eff }}^{\mathrm{o}}(\lambda)$ is the effective index of the fundamental mode, $n_{\text {eff }}^{i}(\lambda)$ is the effective index of the $i$ th propagated modes and $\Lambda$ is the grating period. In a FBG the period is in the same order as the optical wavelength and it follows that only coupling to the backward propagated fundamental mode such that $n_{\text {eff }}^{i}(\lambda)=$ $-n_{\text {eff }}^{\mathrm{o}}(\lambda)$ is allowed. The FBG behaves like a wavelength selective mirror at the phase matching wavelength. In a LPG the period could range from $0.1 \mathrm{~mm}$ to about $1 \mathrm{~mm}$ and it follows that only coupling to forward propagated modes in the cladding is allowed. Because the cladding modes are rapidly attenuated, a typical transmission spectrum for such a fibre will show a number of attenuation bands. If the grating has a length $L$ and the coupling coefficient for the $i$ th cladding mode is $\kappa_{i}$, the minimum transmission of the attenuation bands (2) is found from [15]:

$$
T_{i}=1-\sin \left(\kappa_{i} L\right) .
$$

Changes in the parameters pertaining to the environment of the fibre grating such as temperature, strain and refractive index of the medium surrounding the fibre cladding could change the grating period and the effective refractive indices, resulting in changes in the phase matching condition that will modify the attenuation bands and their minimum transmission values. Connecting variations to the strain, temperature and environmental refractive index variations supported by the optical fibre is nevertheless quite complex since the variations result from a multiple variables system which does not generally admit a single solution. That is why most of the FBG and LPG sensors need a thermal correction that could be made by using a second FBG or LPG sensor which does not support the parameter of influence.

The proposed sensors require the FBG or the LPG to be coated by a thin palladium layer that will change the phase matching conditions when exposed to gaseous hydrogen and potentially promote the coupling of the cladding modes with surface plasmon or evanescent waves. In this configuration, a wavelength shift in the attenuation spectrum is the expected response of the sensor. Palladium has the property of absorbing up to 900 times its volume in hydrogen at room temperature and atmospheric pressure leading to the formation of the hydride $\mathrm{PdH}_{x}$ (where $x$ is the atomic ratio H/Pd). The physical properties of the palladium-hydrogen system are strongly dependent on the gaseous hydrogen pressure and temperature. They are characterized by the existence of two different crystallographic phases called $\alpha$ and $\beta$, separated by a phase transition. Earlier studies [17] have shown that in the $\alpha$-phase, which also corresponds to the crystallographic phase of pure palladium, hydrogen is only slightly absorbed by palladium. In contrast, in the $\beta$-phase, the absorption of hydrogen in palladium is enhanced. The phase transition is characterized by fast changes in the absorption properties of hydrogen in palladium. Following the palladium-hydrogen system properties [17], the response and response time of the sensor are functions of gaseous hydrogen pressure and temperature. Previous studies [12] have shown that due to the weak absorption of hydrogen in palladium, weak responses and short response times are expected in the $\alpha$-phase. In contrast, and due to the enhancement of the absorption of hydrogen in palladium, stronger and saturated responses, independent of the hydrogen concentration, are expected in the $\beta$-phase. These responses are also expected in a longer response time which could exceed $1 \mathrm{~h}$ depending on the temperature. Furthermore, preliminary studies [12] have also shown that the phase transition has an important influence on the repeatability of the performances. Depending on the final processing of the data obtained from the sensor, it is therefore necessary to ensure operating conditions of the sensor either in the $\alpha$-phase or in the $\beta$-phase to keep repeatable performances and to avoid as much as possible operation in the phase transition.

\section{Experimental details}

FBG and LPG were written into Corning SMF $28^{\mathrm{TM}}$ optical fibre whose photosensitivity was enhanced by hydrogen loading. A UV laser was used with a phase mask to write FBG (short periods) into the fibre core after removal of the jacket. LPG were written either by using arc discharges or a UV laser light focused through a $100 \mathrm{~mm}$ focal cylindrical lens. The periods were $0.5 \mu \mathrm{m}$ for the FBG and 200 to $700 \mu \mathrm{m}$ for the LPG. All the grating lengths were in the order of $20 \mathrm{~mm}$ (determined by the number of periods effectively done). Each resulting FBG had a sharp attenuation band at a resonant wavelength of the order of $1550 \mathrm{~nm}$ corresponding to the backward propagated fundamental mode. Each LPG had several larger attenuation bands at resonant wavelengths varying mainly from 1300 to $1600 \mathrm{~nm}$. These attenuation bands correspond to the forward propagated cladding modes.

Having completed the fabrication of the FBG and LPG, the palladium coating was applied by thermal evaporation under vacuum to the region where the jacket had been removed previously. The palladium layer was only applied on one side of the fibre through a $3 \mathrm{~cm}$ length mask centred on the grating. The layer thicknesses were monitored by a quartz crystal sensor. Layer thicknesses of 25-400 nm were realized.

The experimental set-up to measure the sensitivity of the sensors is described in figure 1. It comprises a set of flow 


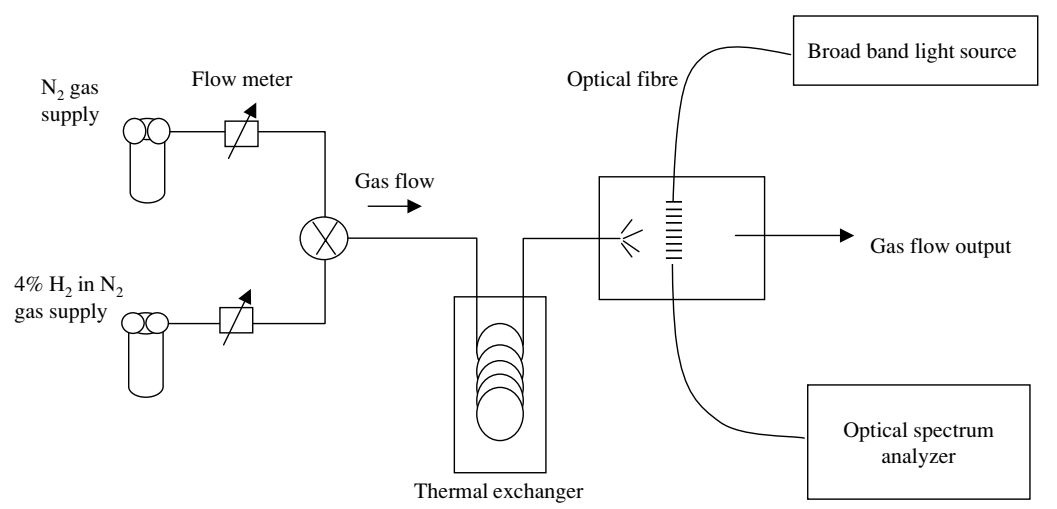

Figure 1. Experimental set-up.
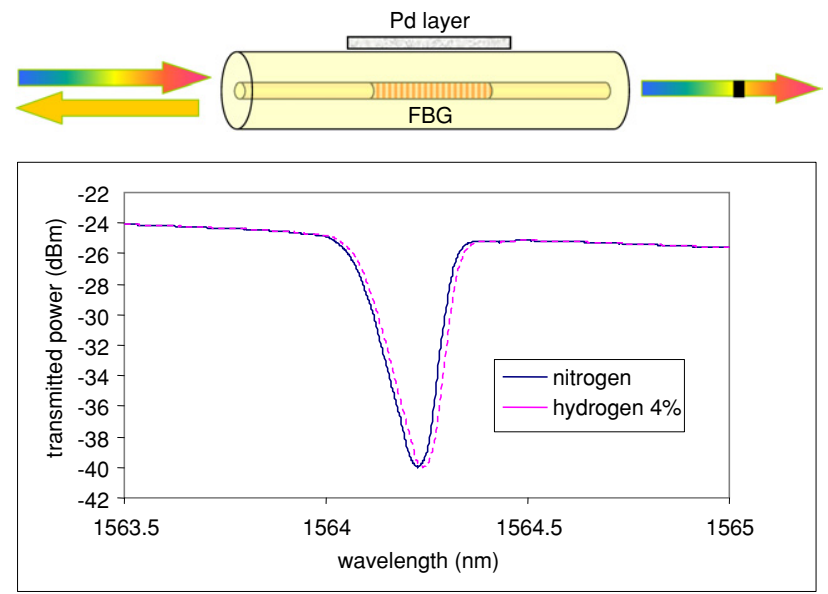

Figure 2. Typical attenuation spectrum for a $0.5 \mu \mathrm{m}$ period FBG under exposure to pure nitrogen and $4 \%$ hydrogen mixture at room temperature.

meters and a thermal exchanger in which either pure nitrogen or a preset mixture of $4 \%$ hydrogen (lower explosive limit) in pure nitrogen is injected with a gas flow set to $500 \mathrm{Nl} \mathrm{h}^{-1}$. The thermally regulated gas is delivered to a gas flow tube into which a FBG or LPG sensor is housed. The fibre is illuminated by a broadband light source and the experimental data are recorded using an optical spectrum analyser. The attenuation bands have been observed for various temperatures between $0{ }^{\circ} \mathrm{C}$ and $40{ }^{\circ} \mathrm{C}$. Due to the strain following the asymmetry of the palladium layer deposition process, small picometric shifts in the attenuation bands have been observed after deposition.

\section{Results and discussion}

Considering the FBG sensor, in the absence of cladding modes and the fundamental mode being strongly confined into the fibre core no major influence on the refractive effective indices was expected due to the palladium layer. Figure 2 shows the typical shape of the attenuation curves obtained with a $50 \mathrm{~nm}$ thick palladium layer when exposed to either pure nitrogen (continuous curve) or a $4 \%$ hydrogen mixture (dotted curve) at room temperature. A positive wavelength shift in the order of $+14 \mathrm{pm}$ towards the upper wavelengths was
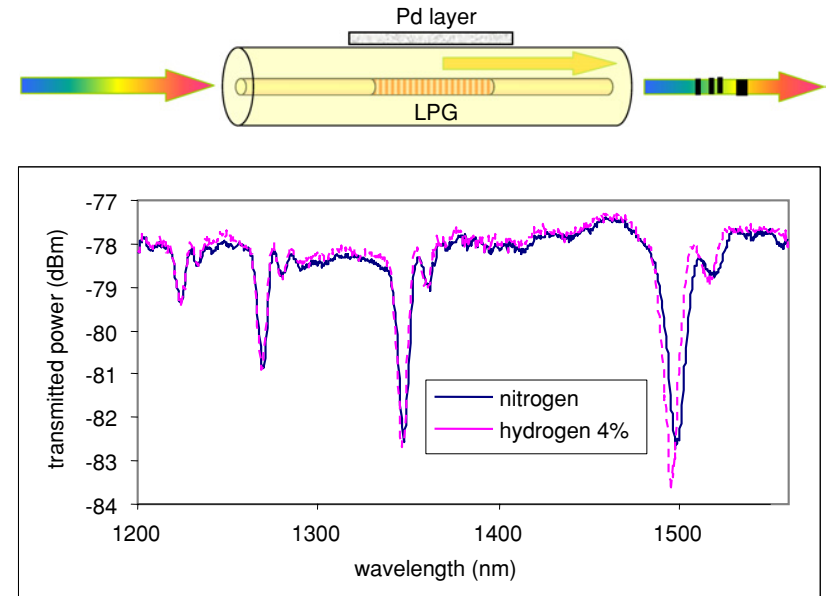

Figure 3. Typical attenuation spectrum for a $400 \mu \mathrm{m}$ period LPG under exposure to pure nitrogen and $4 \%$ hydrogen mixture at room temperature.

observed. This small shift could be explained by the strain following the deformation (growing) of the palladium layer when absorbing hydrogen. From experiments performed at various temperatures the thermal sensitivity was found to be close to $+12 \mathrm{pm}{ }^{\circ} \mathrm{C}^{-1}$. It follows that under such conditions a $4 \%$ hydrogen exposure at room temperature leads to the same response as a $+1{ }^{\circ} \mathrm{C}$ temperature variation. It is, therefore, necessary to take into account the gas temperature variations in the operation of such a sensor. Furthermore, transmission and reflection measurements have been performed without any influence on the sensitivity.

Considering the LPG sensors, the presence of cladding modes leads us to expect refractive index variations due to the palladium layer and furthermore coupling between cladding modes and the surface plasmon wave at the palladium layer's outer interface. Figure 3 shows the typical shape of the attenuation curves obtained with a $50 \mathrm{~nm}$ thick palladium layer when exposed to either pure nitrogen (continuous curve) or a $4 \%$ hydrogen mixture (dotted curve) at room temperature. Negative wavelength shifts, opposite to pure strain effects, in the order of -5 to $-7 \mathrm{~nm}$ towards the lower wavelengths were obtained. The wavelength shift is different for each mode mainly depending on the modal field shape and its overlapping with the surface plasmon mode. It also depends 
on both the grating period and the palladium layer's thickness. Following such results, the sensitivity appears up to 500 times higher than that of the FBG sensors with a better sensitivity for the fundamental mode. From experiments performed at various temperatures the thermal sensitivity was found in the range between $+30 \mathrm{pm}{ }^{\circ} \mathrm{C}^{-1}$ and $+100 \mathrm{pm}{ }^{\circ} \mathrm{C}^{-1}$ depending on both the grating period and the considered mode. It follows that under such conditions a $4 \%$ hydrogen exposure at room temperature leads to the same wavelength shift as a $-50{ }^{\circ} \mathrm{C}$ temperature variation. It is, therefore, not always necessary to take into account the gas temperature variations in the operation of the LPG sensor when operated close to room temperature. No particular influence on the above-mentioned sensing parameters has been observed when using LPG made by either UV exposure or arc discharge.

The response time of the optical spectrum analyser used being too long to perform accurate response time measurements for the sensors, preset exposure times have been chosen to be superior to the response times found by ourselves in the configuration of $[12,13]$ at any studied temperature. $1,2,5$ and $10 \mathrm{~min}$ exposure times to hydrogen were set before each record to ensure that saturated responses were achieved. Saturated, reversible and reproducible responses were observed after $2 \mathrm{~min}$ in any case. Problems of reproducibility have been observed below 2 min but they could be attributed to averaging problems in connection with the data acquisition system.

Non-reproducible results have been observed for palladium layer thicknesses smaller than $40 \mathrm{~nm}$. They could be attributed to a lack of uniformity in the layers for such small thicknesses. In both FBG and LPG configurations, the wavelength shifts could be potentially enhanced by increasing the palladium layer's thickness. Nevertheless, no major influence of the palladium layer thickness has been found in the range $40-100 \mathrm{~nm}$. A slight dependence of the saturated response with the palladium layer's thickness has been observed for the thicker layers (more than $100 \mathrm{~nm}$ ). It shows that the enhancement of the saturated response with the palladium layer's thickness is followed by an increase in the response time. Furthermore, delamination was observed with layers of thicknesses much greater than $100 \mathrm{~nm}$. The delamination of the palladium layer could appear a couple of hours after the first exposure to gases and after several exposure cycles from pure nitrogen to hydrogen-nitrogen mixture. No delamination effects have been observed with thicknesses lower than $150 \mathrm{~nm}$ for several days and weeks for most of the samples.

Further experimental results have been obtained using an in-fibre Mach-Zehnder interferometer made of in-series LPG (figure 4). Such a device is expected to show interesting potentialities towards both the enhancement of the sensitivity and mainly the temperature dependence of the LPG sensors. Preliminary results obtained with two $400 \mathrm{~nm}$ period and $2 \mathrm{~cm}$ length LPG separated by a distance close to $2 \mathrm{~cm}$ have shown that the thermal sensitivity was found in a range between $+30 \mathrm{pm}{ }^{\circ} \mathrm{C}^{-1}$ and $+70 \mathrm{pm}^{\circ} \mathrm{C}^{-1}$ very close to that of a single LPG, but with no influence of the temperature variations on the interfringes. Some preliminary results have also shown that no enhancement of the sensitivity was observed. The influence of parameters such as grating length and period,

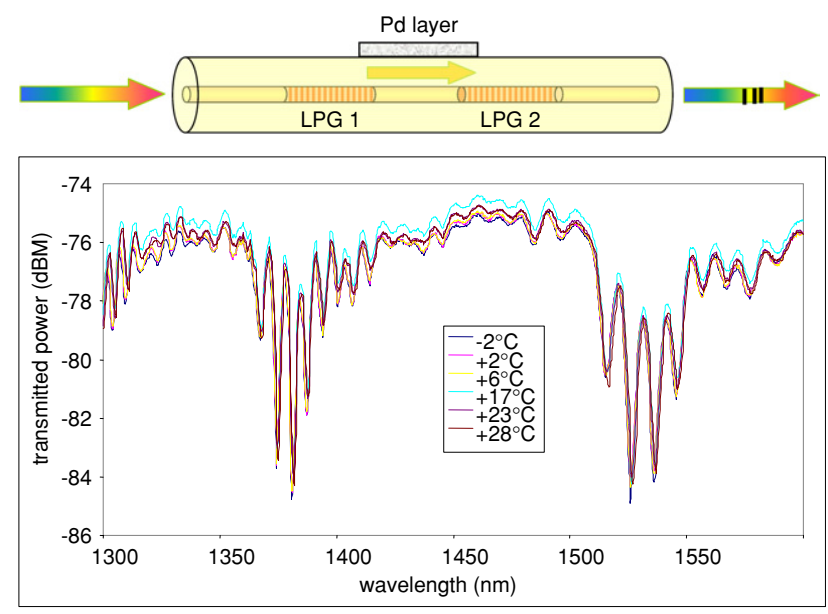

Figure 4. Typical attenuation spectrum for a couple of $400 \mu \mathrm{m}$ period in-series LPG versus temperature.

distance between the two LPG must be considered to confirm and interpret these results. The positioning of the palladium layer (over one or two LPG, over the bare fibre between the LPG or over the entire Mach-Zehnder structure) must also be considered.

\section{Conclusion}

Optical fibre sensors based on FBG and LPG coated by palladium nanolayers have been presented. It has been shown that both techniques could be used for hydrogen sensing but with a sensitivity enhanced by a factor up to 500 when using a LPG sensor. FBG sensors appear to be pure strain sensors using the mechanical deformations of the palladium layer when absorbing hydrogen. LPG sensors are mainly based on the coupling between the cladding modes and evanescent waves or surface plasmon at the interface between the palladium coating and the outer medium. The response of the LPG sensors being stronger than the thermal sensitivity, it has been shown that compensation for the temperature variation may not always be necessary in that case. Preliminary results obtained with an in-fibre Mach-Zehnder interferometer configuration with in-series LPG sensors have also been presented. They show potential interest to compensate for the thermal sensitivity of the fibre gratings.

\section{Acknowledgment}

The authors acknowledge Dr Abdelrafik Malki from the Laboratory of Electronics, Microtechnology and Instrumentation at the University of Rouen, France, who provided us with LPG made by arc discharge.

\section{References}

[1] Hunter G W 1992 A survey and analysis of commercially available hydrogen sensors NASA Technical Memorandum 105878 
[2] Barnes H C and Makel B B 1995 Quantitative leak detection using microelectronic hydrogen sensors 31 st AIAA/ASME/ SAE/ASEE Joint Propulsion Conf. and Exhibit (San Diego, CA) 10-12 July

[3] Alder-Golden M S et al 1992 Laser Raman sensor for measurement of trace-hydrogen gas Appl. Opt. 31-6 831-5

[4] de Groot W A 1995 Fiber-optics based compact gas leak detection system 31 st IAA/ASME/SAE/ASEE Joint Propulsion Conf. and Exhibit (San Diego, CA) 10-12 July

[5] Wyrzykowski K et al 1989 Optical reflection and transmission of PdHx thin films J. Condens. Matter 1 2269-77

[6] Chadwick B et al 1994 A hydrogen sensor based on the optical generation of surface plasmons in palladium Sensors Actuators B 17 215-20

[7] Ito K and Ohgami T 1992 Hydrogen detection based on coloration of anodic tungsten oxide film Appl. Phys. Lett. 60 938-40

[8] Hamagami J et al 1993 Preparation and characterization of an optically detectable $\mathrm{H}_{2}$ gas sensor consisting of $\mathrm{Pd} / \mathrm{MoO}_{3}$ thin films Sensors Actuators B 13-14 281-3
[9] Bearzotti A et al 1992 Integrated optic sensor for the detection of $\mathrm{H}_{2}$ concentrations Sensors Actuators B 7 685-8

[10] Butler M A 1984 Optical fiber hydrogen sensor Appl. Phys. Lett. 45 1007-8

[11] Butler M A 1991 Fiber optic sensor for hydrogen concentration near the explosive limit J. Elec. Soc. 138-9 46-7

[12] Bévenot X et al 2000 Hydrogen leak detection using an optical fibre sensor for aerospace applications Sensors Actuators B 67 57-67

[13] Bévenot X et al 2002 Surface plasmon resonance hydrogen sensor using an optical fibre Meas. Sci. Technol. 13 118-24

[14] Mignani A G 2004 Thoughts on the future for chemical sensing SPIE Proc. $\mathbf{5 5 0 2} 59$

[15] James S W et al 2003 Optical fibre long-period grating sensors: characteristics and application Meas. Sci. Technol. 14 R49-R61

[16] Falciai R et al 2001 Long period gratings as solution concentration sensors Sensors Actuators B 74 74-7

[17] Lewis F A 1967 The Palladium Hydrogen System (New York: Academic) 